Aim of the study: One of the critical steps in molecular oncology diagnostics is obtaining high quality genomic DNA. Therefore, it is important to evaluate and compare the techniques used to extract DNA from tissue samples. Since formalin-fixed, paraffin-embedded (FFPE) tissues are routinely used for both retrospective and prospective studies, we compared three commercially available methods of nucleic acid extraction in terms of quantity and quality of isolated DNA. Material and methods: Slides prepared from 42 FFPE blocks were macro-dissected. Resulting material was divided and processed simultaneously using three extraction kits: QIAamp DNA FFPE Tissue Kit (QIAGEN), Cobas DNA Sample Preparation Kit (Roche Molecular Systems) and Maxwell 16 FFPE Plus LEV DNA Purification Kit (Promega). Subsequently, quantity and quality of obtained DNA samples were analysed spectrophotometrically (NanoDrop 2000, Thermo Scientific). Results of quantitative analysis were confirmed by a fluorometric procedure (Qubit 3.0 Fluorometer, Life Technologies).

Results: The results demonstrated that the yields of total DNA extracted using either Maxwell or Cobas methods were significantly higher compared to the QIAamp method $(p<0.001)$. The Maxwell Extraction Kit delivered DNA samples of the highest quality $(p<0.01)$. However, the highest total yield of extracted DNA was achieved with the Cobas technique, which may be due to a higher volume of eluate compared to the Maxwell method.

Conclusions: To our knowledge, this is the first paper which directly compares three extraction methods: Cobas, Maxwell and QIAamp. The data herein provide information required for the selection of a protocol that best suits the needs of the overal study design in terms of the quantity and quality of the extracted DNA.

Key words: FFPE tissue samples, DNA extraction, Maxwell, Qiagen, Cobas, DNA quality.

Contemp Oncol (Pozn) 2019; 23 (1): 52-58 DOI: https://doi.org/10.5114/wo.2019.83875

\section{DNA extraction from FFPE tissue samples - a comparison of three procedures}

\author{
Agnieszka K. Sarnecka ${ }^{1}$, Dominika Nawrat ${ }^{1}$, Monika Piwowar ${ }^{2}$ \\ Janusz Ligęza ${ }^{3}$, Jakub Swadźba ${ }^{4}$, Piotr Wójcik ${ }^{1}$
}

\section{${ }^{1}$ Oncogene Diagnostics, Krakow, Poland}

2Department of Bioinformatics and Telemedicine, Jagiellonian University Medical College, Krakow, Poland

${ }^{3}$ Division of Biochemistry, Faculty of Medicine and Health Sciences, Andrzej Frycz Modrzewski Krakow University, Krakow, Poland

${ }^{4}$ Department of Laboratory Medicine, Faculty of Medicine and Health Sciences, Andrzej Frycz Modrzewski Krakow University, Krakow, Poland

\section{Introduction}

Recent years have brought us great advances in medical diagnostics in the form of technologies such as next-generation sequencing and gene expression assays. This technological progress contributed to the increased involvement of personal medicine in many clinical areas, including anti-cancer therapies. Identification of the mutation predisposing the patient to the particular treatment option is a key factor impacting the result of the therapy. Therefore, molecular oncological diagnostics is one of the fastest growing areas of laboratory medicine.

The critical step of every diagnostic procedure is the preparation and isolation of high quality starting material [1]. This requires optimization of both sample collection and preservation methods. As there is no gold standard method for tissue sample fixation, heterogeneity of techniques used for sample preservation impedes the following preparative and diagnostic procedures [2]. Moreover, most diagnostic laboratories analyse material from different clinical centres and even small variations in standard fixation protocols may result in large differences in quality and quantity of extracted DNA.

The most frequently used method of tissue sample preservation for medical applications is formaldehyde fixation followed by paraffin embedding. On the one hand it allows one to preserve tissue architecture, cell shape and the components of the cell (proteins, carbohydrates, etc.). On the other hand, prolonged formalin fixation causes the crosslinking of proteins and nucleic acids and random breakages in nucleotide sequences [3-6].

In the present study we compared three kits for formalin-fixed, paraffin-embedded (FFPE) DNA extraction. Two of them use a method based on binding of DNA to either a silica membrane (QIAamp DNA FFPE Tissue Kit) or a glass fibre filter insert (Cobas DNA Sample Preparation Kit, Roche). The third one, automated Maxwell 16 FFPE Plus LEV DNA Purification Kit (Promega), utilizes silica-clad paramagnetic particles (PMPs) for transfer of genetic material through/purifying reagents in the cartridge into the elution buffer (nuclease-free water). To the best of our knowledge, this is the first direct comparison of these methods.

\section{Material and methods}

\section{Sample preparation}

Archived tissue samples included in the present study were submitted by different pathology laboratories to Oncogene Diagnostics for the evaluation of mutational status. All analysed samples were obtained during routine 
diagnostic procedures. All patients' personal data were anonymized, and no information on the identity of any individual was available. A total of 42 FFPE tissues (including melanomas, colon cancer and lung adenocarcinomas) were dewaxed/deparaffinized (the procedure included 3 washes in xylene for 3 minutes followed by 3 washes in 99.8\% ethanol for 3 minutes) and sliced into $5 \mu \mathrm{m}$ tissue sections. Tumour-rich regions of interest were identified and marked on the H\&E stained slides and confirmed by a pathologist to decrease the risk of false negative results. Tissue material was macrodissected from unstained slides using $H \& E$ as a guide. Equal amounts (the same number of paraffin sections and area on the slides) of sections were macrodissected and subjected to each of the extraction procedures. The scheme summarizing extraction protocols can be found in Figure 1.

Prior to the implementation of a specific procedure all samples were treated equally. Furthermore, to avoid the variation associated with the different interpretation of the selected tissue fragments, all slides were macrodissected by one person.

\section{DNA extraction}

All procedures were carried out according to the manufacturer's instructions. RNase was only used with the QIAamp DNA FFPE Tissue Kit (Fig. 1). In the case of two methods we used an additional incubation of samples at $90^{\circ} \mathrm{C}$ to reverse cross-linkages caused by formalin fixation (mentioned methods are indicated in Figure 1 with asterisks). The Maxwell method was the only one with a lower volume of the DNA extraction buffer.

\section{DNA quantification}

The concentration of DNA in all obtained samples was evaluated using two methods: spectrophotometric measurement of absorbance at $260 \mathrm{~nm}$ wavelength (NanoDrop 2000, Thermo Scientific, Wilmington, DE) and the fluorometric method based on binding of double-stranded DNA (dsDNA)-selective fluorescent dyes (dsDNA) (Qubit 3.0 Fluorometer/Life Technologies, Invitrogen).

\section{Evaluation of quantity and quality of extracted DNA}

The quality of extracted DNA was evaluated using an absorbance ratio of $260 \mathrm{~nm}$ to $280 \mathrm{~nm}\left(\mathrm{~A}_{260} / \mathrm{A}_{280}\right)$. Samples with the $A_{260} / A_{280}$ ratio falling within the range of 1.8-2.0 were considered to be of good quality. Subsequent Qubit analysis allowed the assessment of the dsDNA content in the isolated samples.

\section{Statistics}

Statistical analysis was performed with the R software (release 3.3.2) [7], corrplot [8] and PerformanceAnalytics libraries [9] for visualizations and the doBy library [10] for descriptive statistics. The differences in the quantity and purity between extracted DNA samples were evaluated with the Kruskal-Wallis one-way ANOVA test by ranks. Dunn test with the Holm-Sidak adjustment from the Dunn test library [11] was used as a post-hoc test. Statistica 12.5 was used for data visualisation.

\section{Results}

We compared the quantity and purity of samples extracted with three different DNA isolation methods: Cobas, Maxwell, and QIAamp. The results of spectrophotometric and fluorimetric analysis of nucleic acid content in extracted samples are shown in Figure 2. A summary of the statistical analysis is presented in Table 1 .

Maxwell and Cobas turned out to be the most efficient methods in terms of DNA concentration in the extracted samples. The analysis with the NanoDrop spectrophotometer showed average concentration of nucleic acids of $50.60 \mathrm{ng} / \mu \mathrm{l}$ for Cobas and $102.72 \mathrm{ng} / \mu \mathrm{l}$ for the Maxwell method ( $p>0.05$ ). The average concentration of nucleic acids in samples extracted with QIAamp was 60\% lower in comparison to Cobas and over $80 \%$ lower in comparison to Maxwell $(p<0.0001)$.

Fluorimetric analysis of samples with the Qubit method showed similar differences in the outcomes of the investigated DNA isolation techniques. However, the average

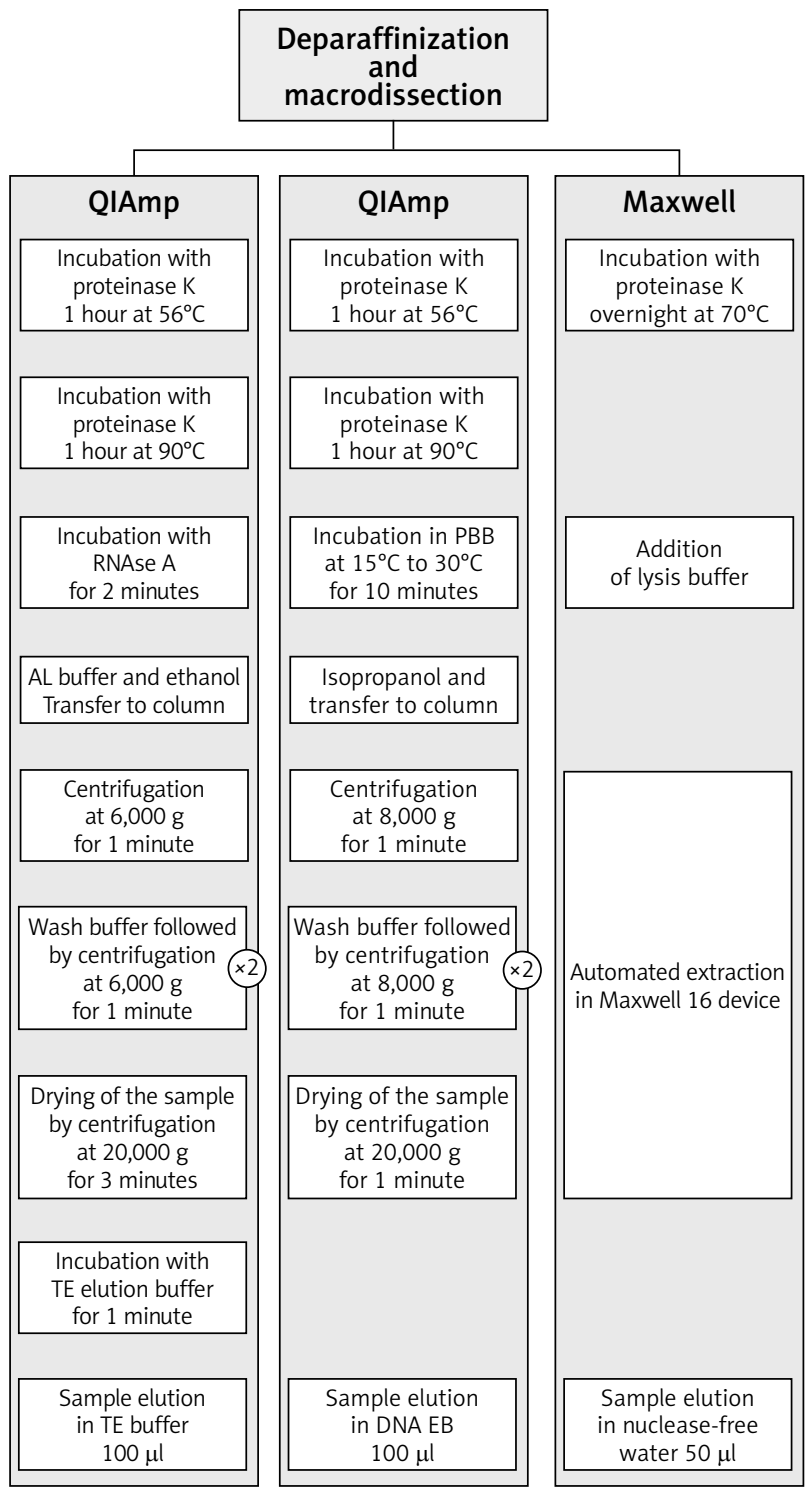

Fig. 1. Scheme of the DNA isolation procedures 

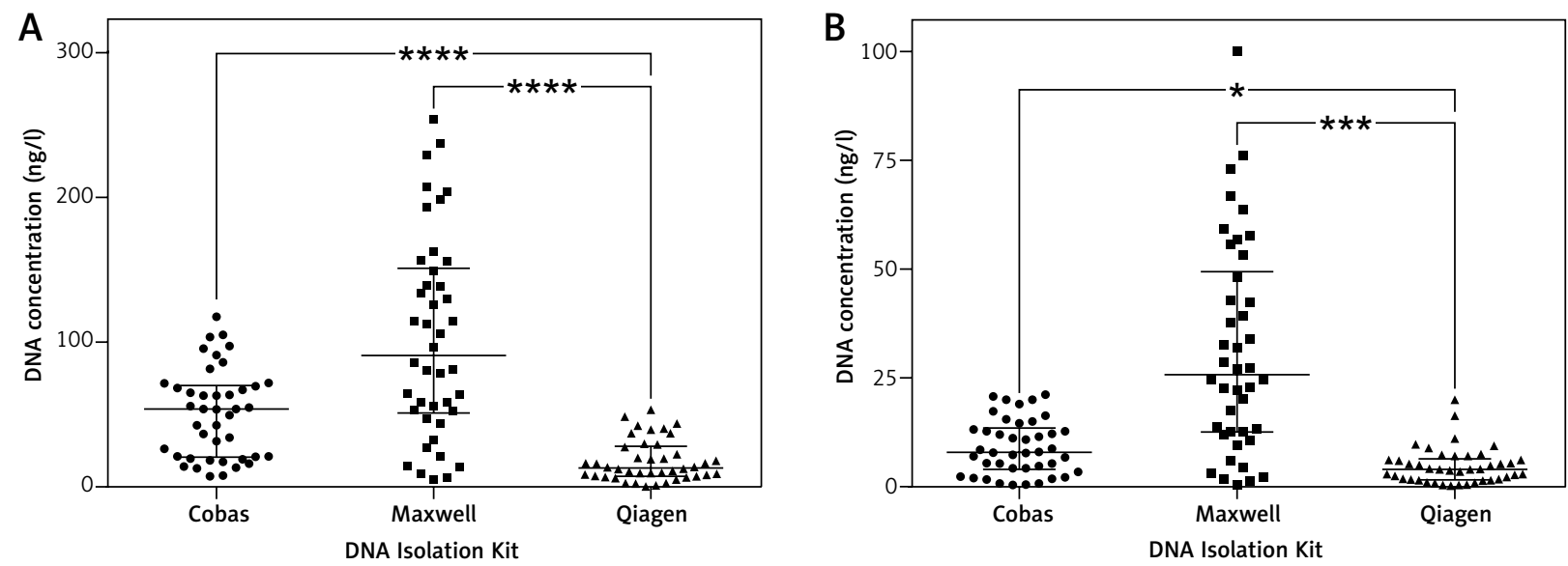

Fig. 2. Quantitative analysis of DNA concentration in samples isolated from FFPE specimens. A) Spectrophotometric analysis with NanoDrop. B) Fluorimetric analysis with Qubit method. Results are presented as median and interquartile range $(\mathrm{IQR}){ }^{*} p<0.05 ;{ }^{* *} p<0.01 ;{ }^{* * *} p<0.001$; ${ }^{* * *} p<0.0001$

Table 1. Results of qualitative and quantitative analysis of DNA samples isolated using Cobas (C), Maxwell (M) and QIAamp (Q) FFPE DNA extraction kits

\begin{tabular}{|c|c|c|c|c|c|c|c|}
\hline \multirow{2}{*}{$\begin{array}{l}\text { Measuring } \\
\text { device }\end{array}$} & \multirow[t]{2}{*}{ Parameter } & \multirow[t]{2}{*}{ Method } & \multirow[t]{2}{*}{ Mean } & \multirow[t]{2}{*}{ Median } & \multirow[t]{2}{*}{ Range } & \multicolumn{2}{|c|}{$p$-value } \\
\hline & & & & & & Kruskal-Wallis & post-hoc: Dunn test \\
\hline \multirow{6}{*}{ NanoDrop } & \multirow{3}{*}{ Quantity (ng/ul) } & Cobas & 50.60 & 53.75 & $7.3-117.5$ & \multirow{3}{*}{$2.101 \mathrm{e}-08$} & C vs. M: 0.4693 \\
\hline & & Maxwell & 102.72 & 90.95 & $5.30-253.90$ & & C vs. Q: 0.0000 \\
\hline & & QIAamp & 18.00 & 13.20 & $0.3-53.4$ & & M vs. Q: 0.0000 \\
\hline & \multirow{3}{*}{ Purity $\left(\mathrm{A}_{260} / \mathrm{A}_{280}\right)$} & Cobas & 1.84 & 1.77 & $1.37-3.61$ & \multirow{3}{*}{0.00046} & C vs. M: 0.2182 \\
\hline & & Maxwell & 1.82 & 1.81 & $1.36-2.53$ & & C vs. Q: 0.0003 \\
\hline & & QIAamp & 1.78 & 1.90 & $-5.09-4.36$ & & M vs. Q: 0.0033 \\
\hline \multirow{3}{*}{ Qubit } & \multirow{3}{*}{ Quantity (ng/ul) } & Cobas & 9.15 & 7.92 & $0.43-21.2$ & \multirow{3}{*}{0.00185} & C vs. M: 0.1802 \\
\hline & & Maxwell & 31.28 & 25.80 & $0.49-100.0$ & & C vs. Q: 0.0009 \\
\hline & & QIAamp & 4.79 & 4.04 & $0.31-20.0$ & & M vs. Q: 0.0120 \\
\hline
\end{tabular}

concentrations obtained with this method were much lower, i.e. $9.15 \mathrm{ng} / \mu \mathrm{l}, 31.28 \mathrm{ng} / \mu \mathrm{l}$ and $4.79 \mathrm{ng} / \mu \mathrm{l}$ for the Cobas, Maxwell and QIAamp method respectively.

Both spectrophotometric and fluorometric measurements showed significant similarities in the DNA concentration in the samples obtained with the Maxwell and Cobas kits (Fig. 3), with Spearman correlation coefficient of 0.85 and 0.77 for the NanoDrop and Qubit instrument respectively. The results of QIAamp extraction correlated with the results of Maxwell and Cobas procedures, but the relationship was weaker.

As mentioned before, extraction of DNA from FFPE samples using the Maxwell method resulted in the highest concentration of DNA in the solutions. However, due to a lower final volume of the samples obtained with the Maxwell method $(50 \mu \mathrm{l})$ as compared to the other two methods $(100 \mu \mathrm{l})$, the total yield of DNA isolated with the Cobas and Maxwell methods was comparable and in both cases much higher than for the QIAamp technique.

The quality of the extracted DNA samples was assessed spectrophotometrically using the ratio of absorbance at $260 \mathrm{~nm}$ and $280 \mathrm{~nm}$ (Table 1). The Maxwell DNA Purification Kit showed the best performance in terms of quality
(Table 2). Twenty-six of 42 analysed samples (62\%) fulfilled the purity criterion, which was $A_{260} / A_{280}$ ratio within the range of 1.8-2.0. Other methods had a lower success rate: 50 and $33 \%$ of samples could be considered of good quality for the QIAamp and Cobas methods respectively. Moreover, the Maxwell method showed the highest aggregation of similar quality data (purity 1.8-2.0 criterion) of all three methods, which can be seen in the graph (Fig. 4).

We also compared the results of DNA quantitative analysis of two implemented methods: NanoDrop and Qubit. The correlation was very strong, with the Spearman correlation coefficient exceeding 0.9 for pooled data (Fig. 5). However, the NanoDrop method gave higher readings of DNA concentration in all analysed samples, as compared to Qubit.

\section{Discussion}

In the present study, the Maxwell method of DNA extraction demonstrated the greatest performance of all compared techniques. The concentration of DNA in samples obtained in the Maxwell isolation was the highest as compared to Cobas and QIAamp, though taking differences in elution volume into account, total yield of extracted 

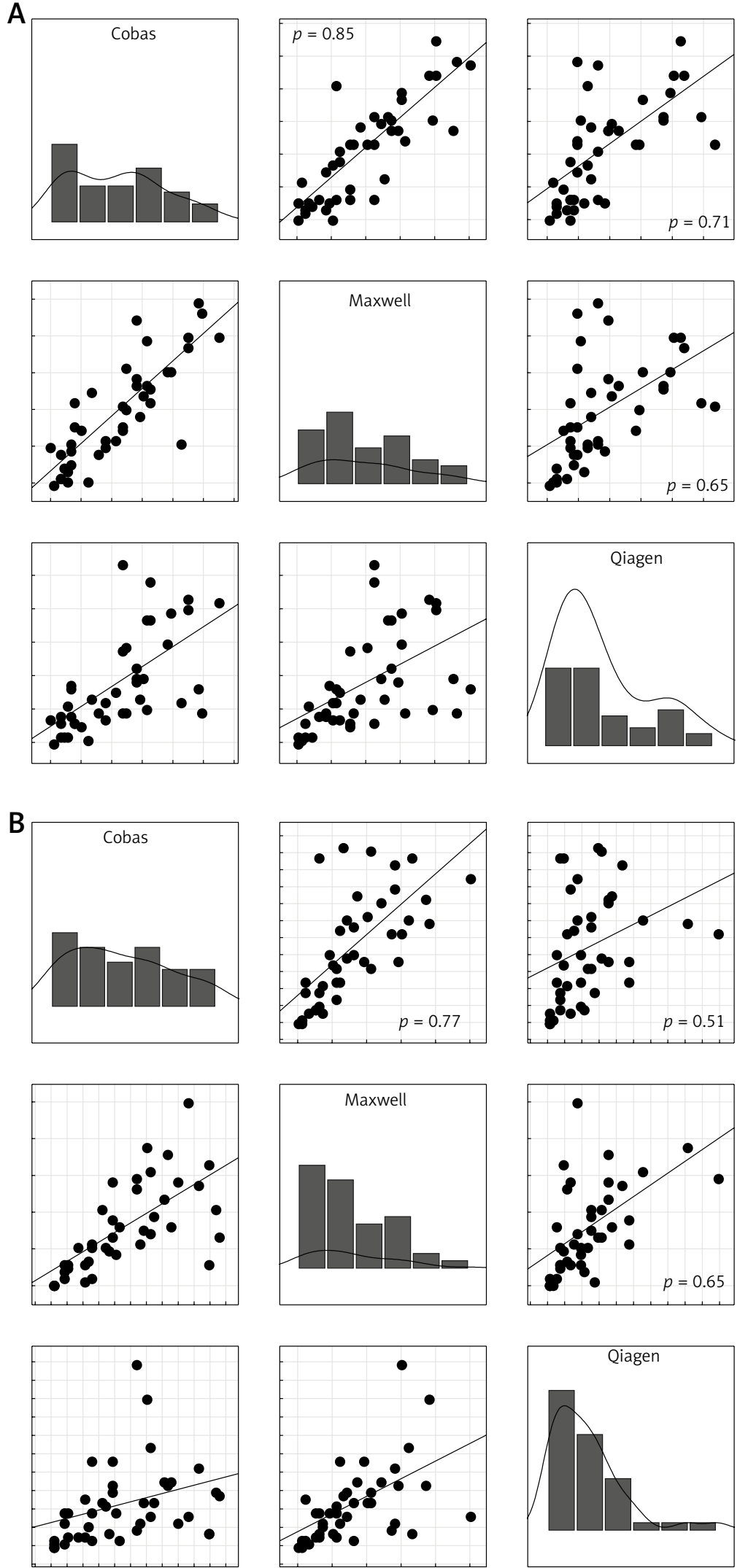

Fig. 3. Correlation of the concentration of DNA samples obtained with Cobas, Maxwell and QIAamp techniques. A) Spectrophotometric analysis with NanoDrop. B) Fluorimetric analysis with Qubit method. Correlations with Spearman coefficient in the range $0.4-0.7$ were considered as moderate and with Spearman coefficient greater than 0.7 were regarded as strong. All correlations were statistically significant with $p<0.0001$
DNA was comparable to that obtained using the Cobas method. The QIAamp method was the least efficient in terms of quantity of the extracted DNA. Moreover, the isolation of DNA with the Maxwell kit resulted in the greatest number of samples that fulfilled the purity criterion $\left(A_{260} / A_{280}\right.$ ratio within a range of 1.8-2.0). The Maxwell method was previously reported by Heydt et al. to be the most efficient of five tested automated methods [12]. Furthermore, fluorometric Qubit analysis showed RNA contamination in only one sample obtained with the Maxwell extraction kit. The isolates acquired with the QIAamp method turned out to be highly contaminated with RNA. The DNA samples obtained with the Cobas method showed less RNA contamination as compared to QIAamp, despite the fact that the QIAamp method implements an RNAseA incubation step. Similar findings had previously been reported by Malthora et al. [13]. High efficiency of DNA extraction and high quality of isolated samples designate the Maxwell technique as the first-choice method for DNA extraction from FFPE tissues, especially for laboratories with high throughput of samples analysed.

Although the Cobas method did not excel in the extraction of DNA from FFPE tissue samples, it proved to be indispensable for isolating DNA from small tissue materials (fine-needle biopsies, etc.). In our experience, DNA isolated from this problematic material using Cobas can be easily amplified. In contrast, the isolates obtained by the Maxwell or QIAamp methods are frequently of poor quality and unsuitable for subsequent analysis (data unpublished). Similar observations were described in the paper of Hu et al. comparing QIAamp and Cobas DNA extraction methods for studies of the EGFR gene mutations in biopsy specimens [14].

The measurement of nucleic acid concentration in the extracted samples is a very important step in diagnostics. The spectrophotometric evaluation is often used because it is easy and inexpensive. Moreover, spectrophotometric measurements provide additional information on the possible contaminants in the analysed sample. On the other hand, there is no possibility to distinguish different nucleic acid species on the basis of sample absorbance alone. The fluorometric methods, based on fluorescent dyes, such as Hoechst 33258 and PicoGreen, are more sensitive and selective. These dyes allow one to detect 10 and 25 pg of double stranded nucleic acid in $1 \mu$ of eluate, respectively. Furthermore, the measurement is not influenced by proteins or other contaminants. The main drawbacks are 
Table 2. Sample distribution frequency according to purity level criterion

$\begin{array}{lccc}\mathrm{A}_{260} / \mathrm{A}_{230} & \mathbf{1 . 8 - 2 . 0} & <\mathbf{1 . 8} & >\mathbf{2 . 0} \\ \text { Cobas } & 14 & 24 & 4 \\ \text { Maxwell } & 26 & 15 & 1 \\ \text { QIAamp } & 21 & 9 & 12\end{array}$

the limitation to the quantitative information only and the higher costs of implementation [15].

In the present study, the DNA concentration measured with the spectrophotometric method (NanoDrop) was 0.98 to 16.60 times higher than concentrations calculated from the fluorometric measurements (2.94 to 16.60 times higher for the Cobas method, 2.05 to 6.72 times higher for the Maxwell method and 0.98 to 10.94 times higher for the QIAamp method). Higher readings of the nucleic acid concentration in the spectrophotometric
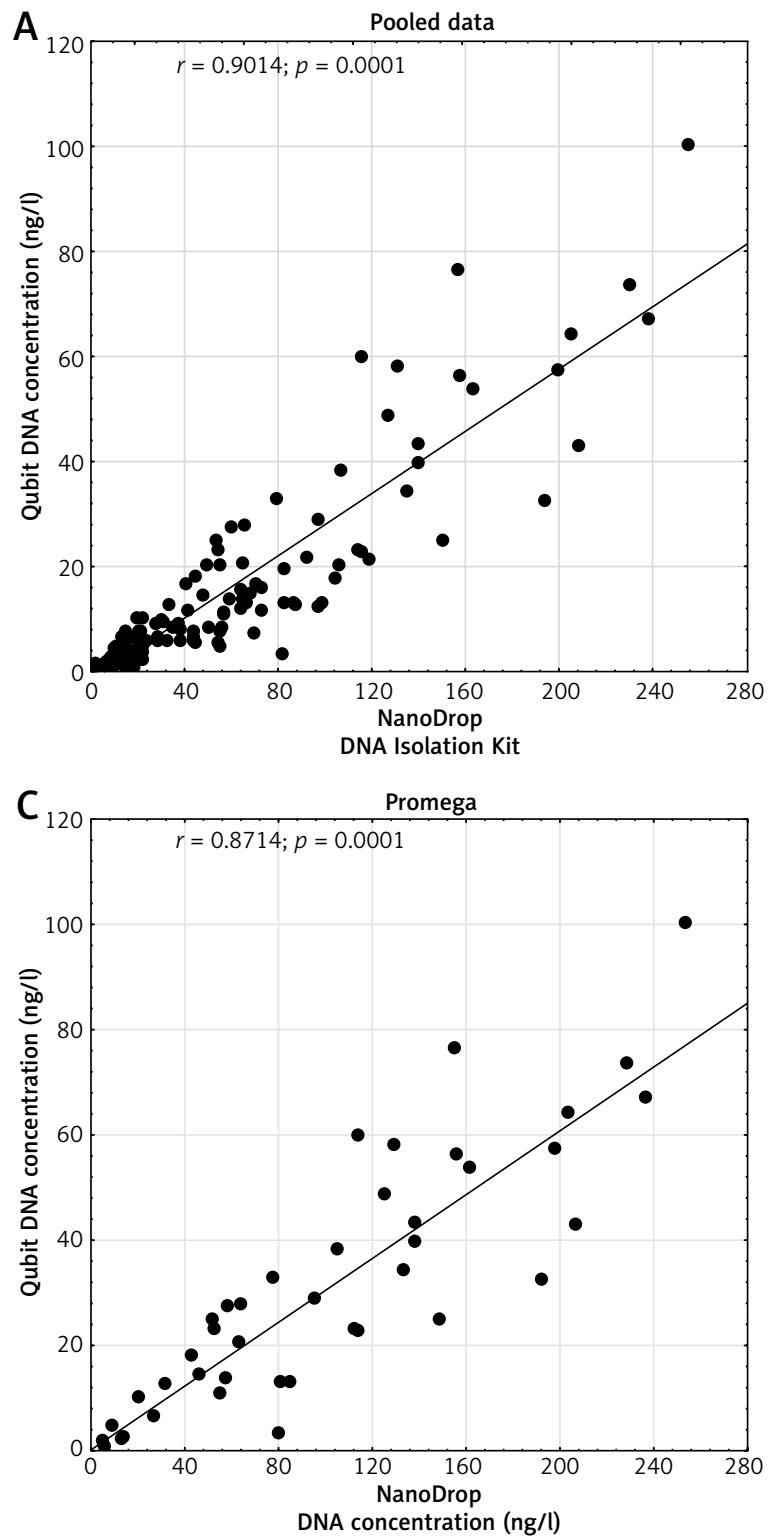

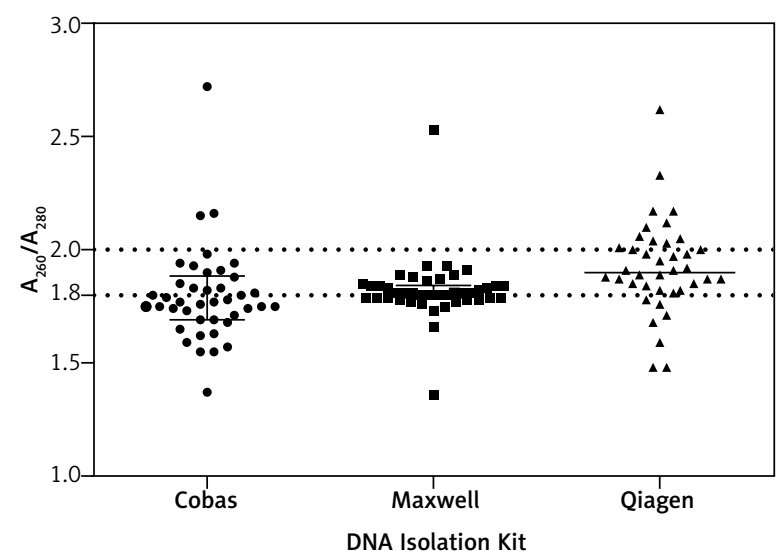

Fig. 4. Spectrophotometric assessment of extracted DNA quality expressed as an absorbance ratio of $260 \mathrm{~nm}$ to $280 \mathrm{~nm}\left(\mathrm{~A}_{260} / \mathrm{A}_{230}\right)$. Median is shown with vertical solid lines. Error bars represent IQR. Dashed lines enclose high quality DNA samples with $A_{260} / A_{230}$ ratio falling within the range 1.8-2.0. $Y$-axis scale was narrowed to the range 1.0-3.0 to make the graph more transparent; therefore, four outlier points are not visible on the graph
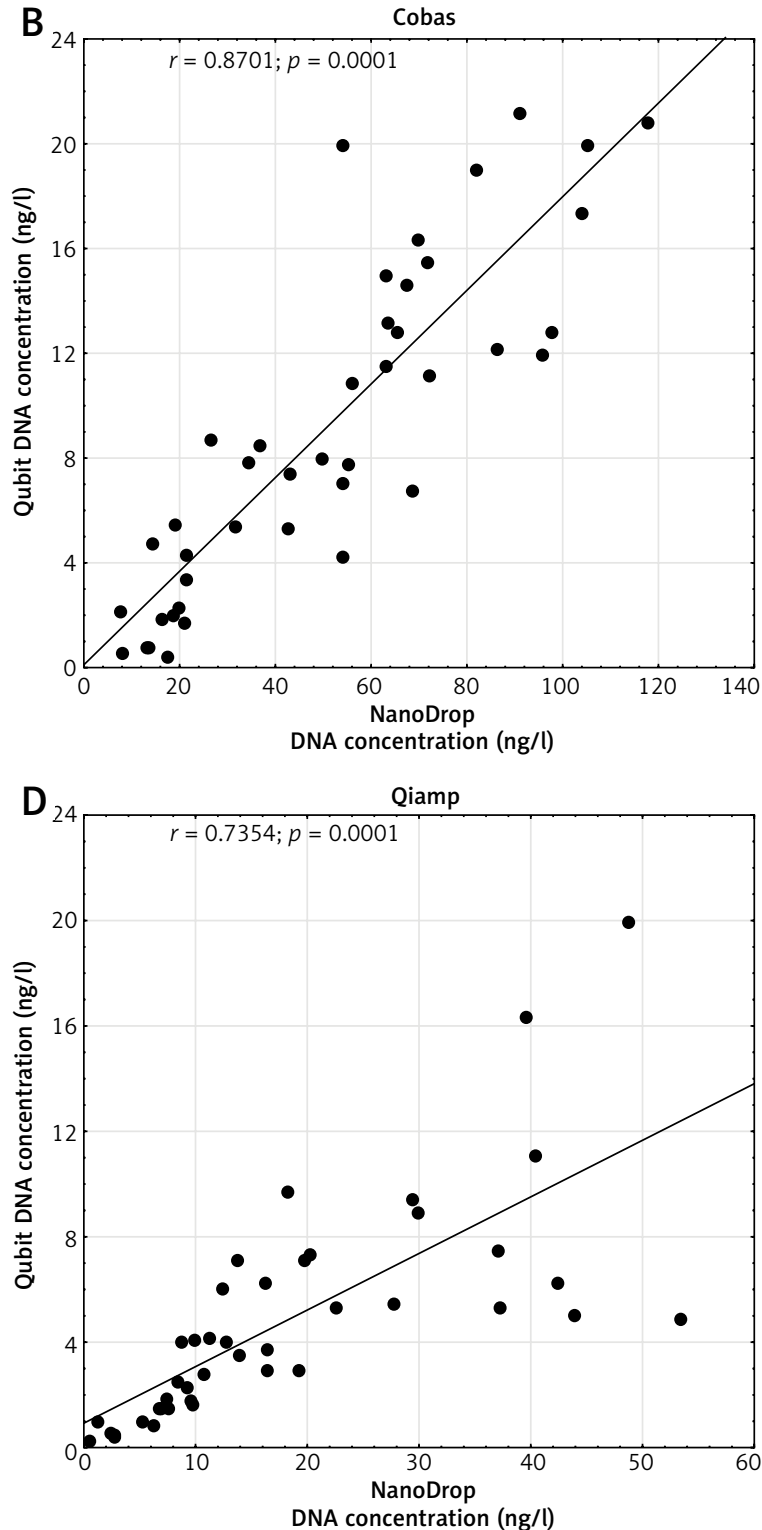

Fig. 5. Correlation of DNA concentration readings between spectrophotometric NanoDrop method and fluorimetric Qubit method 
method (NanoDrop) may arise from the presence of RNA in the isolates or from other impurities absorbing light at $260 \mathrm{~nm}$. Therefore, the fluorometric Qubit method seems to be more accurate due to utilization of dsDNA specific dyes. A note of caution is due here. Although PicoGreen dye is not affected by contaminants, the compromised DNA integrity may impede the measurements [15-17]. There are many factors which may impair DNA in the FFPE tissue specimen. Each step of sample preparation, from collection (cold and warm ischemia, method of decalcification and size of the specimen), through fixation (buffered formalin, time, temperature) to embedding and storage (paraffin reagents, time of storage, humidity), may affect DNA quality, impeding analysis at the further stages of diagnostics [18-24]. Interestingly, quantitative analyses of DNA samples extracted from non-FFPE sources revealed concordance between spectrophotometric and fluorometric methods [25, 26]. Therefore, we conclude that the observed discrepancies between concentrations of DNA samples determined using the NanoDrop and Qubit instruments may be a result of tissue processing during FFPE core preparation. We believe that formalin fragmentation and incomplete deparaffinization are the key factors interfering in the quantitative evaluation of the extracted DNA samples. Moreover, incubation of the tissue specimens at lower temperatures (e.g. $56^{\circ} \mathrm{C}$ ) may result in less efficient reversion of nucleic acid-protein cross-links, manifesting in DNA yield differences between distinct isolation methods [27]. In our experience, fluorometric analysis provides more reliable data for more sensitive molecular methods such as microsatellite instability (MSI) testing or next generation sequencing (NGS) (data unpublished). The NanoDrop measurements tend to be less accurate due to frequent RNA contamination of samples [25].

\section{Conclusions}

The purpose of the current research was to assess and compare the performance of three commonly used FFPE DNA extraction methods. The study is limited by the lack of information on the result of the subsequent molecular analyses, although it does offer an important insight into the influence of preanalytical processing of the archival tissue specimens. We are aware that the mixed origin of the tested FFPE samples may have increased the variance of results. Different pathology laboratories have their own fixing protocols. Moreover, the method of collection and preservation of the tissue may differ between hospitals. Nevertheless, analysis of tissue specimens obtained from different sources makes our study less prone to bias resulting from laboratory-specific deviation from common protocols. Furthermore, the mixed origin of the samples allows more general conclusions to be drawn.

The results of our present study have proved that the Maxwell method provides an advantage in terms of both yield and quality of DNA isolated from FFPE tissue specimens. However, further tests on a larger number of samples are needed to confirm its superior performance. Moreover, additional methods should be included in the design of future studies to confirm suitability of the extracted DNA samples for subsequent molecular diagnostics.

The authors declare no conflict of interest.

\section{References}

1. Ali N, Rampazzo RDCP, Costa ADiT, Krieger MA. Current Nucleic Acid Extraction Methods and Their Implications to Point-of-Care Diagnostics. Biomed Res Int 2017; 2017: 9306564.

2. Donczo B, Guttman A. Biomedical analysis of formalin-fixed, paraffin-embedded tissue samples: The Holy Grail for molecular diagnostics. J Pharm Biomed Anal 2018; 155: 125-134.

3. Williams C, Pontén F, Moberg C, Söderkvist P, Uhlén M, Pontén J, Sitbon $\mathrm{G}$, Lundeberg J. A high frequency of sequence alterations is due to formalin fixation of archival specimens. Am J Pathol 1999; 155: 1467-1471.

4. Srinivasan M, Sedmak D, Jewell S. Effect of fixatives and tissue processing on the content and integrity of nucleic acids. Am J Pathol 2002; 161: 1961-1971.

5. Wong SQ, Li J, Tan AY, et al. Sequence artefacts in a prospective series of formalin-fixed tumours tested for mutations in hotspot regions by massively parallel sequencing. BMC Med Genomics 2014; 7: 23.

6. Do H, Dobrovic A. Sequence artifacts in DNA from formalin-fixed tissues: causes and strategies for minimization. Clin Chem 2015; 61: 64-71.

7. R Core Team (2018). R: A Language and Environment for Statistical Computing. R Foundation for Statistical Computing, Vienna, Austria 2015, 1: 409. http://www.R-project.org/.

8. Wei T, Simko V. Visualization of a Correlation Matrix: Package "corrplot” (Version 0.82), 2016. https://cran.r-project.org/web/packages/corrplot/corrplot.pdf

9. Peterson BG, Carl P. PerformanceAnalytics: Econometric tools for performance and risk analysis. R package version 1.4.3541, 2014. https://cran.r-project.org/web/packages/PerformanceAnalytics/ PerformanceAnalytics.pdf

10. Hojsgaard S, Halekoh U. PerformanceAnalytics: Econometric tools for performance and risk analysis. R package version 1.4.3541, 2015.

11. Dinno A. Dunn's Test of Multiple Comparisons Using Rank Sums. R package version 1.3.2, 2016. https://cran.r-project.org/web/ packages/dunn.test/dunn.test.pdf

12. Heydt C, Fassunke J, Künstlinger $\mathrm{H}$, et al. Comparison of pre-analytical FFPE sample preparation methods and their impact on massively parallel sequencing in routine diagnostics. PLoS One 2014; 9: e104566.

13. Malhotra K, Gulati U, Balzer B, Wu HY. Comparison of DNA EXtraction Methods from Formalin-Fixed, Paraffin- Embedded Tissue and their Impact on Real-Time PCR-Based Mutation Assays. J Med Diagn Meth 2012; 1: 107.

14. Hu Y-C, Zhang Q, Huang Y-H, Liu Y-F, Chen H-L. Comparison of two methods to extract DNA from formalin-fixed, paraffin-embedded tissues and their impact on EGFR mutation detection in non-small cell lung carcinoma. Asian Pac J Cancer Prev 2014; 15: 2733-2737.

15. Sedlackova T, Repiska G, Celec P, Szemes T, Minarik G. Fragmentation of DNA affects the accuracy of the DNA quantitation by the commonly used methods. Biol Proced Online 2013; 15: 5.

16. Georgiou CD, Papapostolou I. Assay for the quantification of intact/fragmented genomic DNA. Anal Biochem 2006; 358: 247-256.

17. Singer VL, Jones LJ, Yue ST, Haugland RP. Characterization of PicoGreen reagent and development of a fluorescence-based solution assay for double-stranded DNA quantitation. Anal Biochem 1997; 249: 228-238.

18. Zsikla V, Baumann M, Cathomas G. Effect of buffered formalin on amplification of DNA from paraffin wax embedded small biopsies using real-time PCR. J Clin Pathol 2004; 57: 654-656. 
19. Turashvili G, Yang W, McKinney S, et al. Nucleic acid quantity and quality from paraffin blocks: defining optimal fixation, processing and DNA/RNA extraction techniques. Exp Mol Pathol 2012; 92: 33-43.

20. Sah S, Chen L, Houghton J, Kemppainen J, Marko AC, Zeigler R, Latham GJ. Functional DNA quantification guides accurate next-generation sequencing mutation detection in formalin-fixed, paraffin-embedded tumor biopsies. Genome Med 2013; 5: 77.

21. Taga M, Eguchi H, Shinohara T, et al. Improved PCR amplification for molecular analysis using DNA from long-term preserved formalin-fixed, paraffin-embedded lung cancer tissue specimens. Int J Clin Exp Pathol 2013; 6: 76-79.

22. Bass BP, Engel KB, Greytak SR, Moore HM. A review of preanalytical factors affecting molecular, protein, and morphological analysis of formalin-fixed, paraffin-embedded (FFPE) tissue: how well do you know your FFPE specimen? Arch Pathol Lab Med 2014; 138: 15201530.

23. Nam SK, Im J, Kwak Y, Han N, Nam KH, Seo AN, Lee HS. Effects of fixation and storage of human tissue samples on nucleic Acid preservation. Korean J Pathol 2014; 48: 36-42.

24. Nechifor-Boilă AC, Loghin A, Vacariu V, Halațiu VB, Borda A. The storage period of the formalin-fixed paraffin-embedded tumor blocks does not influence the concentration and purity of the isolated DNA in a series of 83 renal and thyroid carcinomas. Rom J Morphol Embryol 2015; 56: 759-763.

25. Kapp JR, Diss T, Spicer J, et al. Variation in pre-PCR processing of FFPE samples leads to discrepancies in BRAF and EGFR mutation detection: a diagnostic RING trial. J Clin Pathol 2015; 68: 111-118.

26. Sengüven B, Baris E, Oygur T, Berktas M. Comparison of Methods for the Extraction of DNA from Formalin-Fixed, Paraffin-Embedded Archival Tissues. Int J Med Sci 2014; 11: 494-499.

27. Seiler C, Sharpe A, Barrett JC, Harrington EA, Jones EV, Marshall GB. Nucleic acid extraction from formalin-fixed paraffin-embedded cancer cell line samples: a trade-off between quantity and quality? BMC Clin Pathol 2016; 16: 1-13.

\section{Address for correspondence}

\section{Agnieszka K. Sarnecka}

Oncogene Diagnostics

86 Mogilska St.

31-465 Krakow, Poland

e-mail:asarnecka@oncogene.pl

Submitted: 12.11 .2018

Accepted: $\quad 26.01 .2019$ 